\title{
Expression of the clock gene Rev-erbo in the brain controls the circadian organisation of food intake and locomotor activity, but not daily variations of energy metabolism
}

\author{
S. Sen ${ }^{1,2}$ (1) | S. Dumont ${ }^{1}$ | D. Sage-Ciocca ${ }^{3}$ | S. Reibel ${ }^{3}$ | P. de Goede ${ }^{4}$ | \\ A. Kalsbeek ${ }^{2,4}$ | E. Challet $^{1}$
}

\author{
${ }^{1}$ Regulation of Circadian Clocks Team, \\ Institute of Cellular and Integrative \\ Neurosciences, UPR3212, Centre National de \\ la Recherche Scientifique (CNRS), University \\ of Strasbourg, Strasbourg, France \\ ${ }^{2}$ Hypothalamic Integration Mechanisms, \\ Netherlands Institute for Neuroscience (NIN), \\ Amsterdam, The Netherlands \\ ${ }^{3}$ Chronobiotron, UMS3415, CNRS, University \\ of Strasbourg, Strasbourg, France \\ ${ }^{4}$ Department of Endocrinology and \\ Metabolism, Academic Medical Center \\ (AMC), University of Amsterdam, Amsterdam, \\ The Netherlands

\section{Correspondence} \\ Satish Sen, Regulation of Circadian Clocks \\ Team, Institute of Cellular and Integrative \\ Neurosciences, UPR3212, Centre National de \\ la Recherche Scientifique (CNRS), University \\ of Strasbourg, Strasbourg, France. \\ Email: ssenbiotech14@gmail.com \\ Funding information \\ Neurotime Erasmus Mundus Program; \\ European Doctoral College of University of \\ Strasbourg and Eurometropolis of Strasbourg; \\ Centre National de la Recherche Scientifique, \\ University of Strasbourg; University of \\ Amsterdam
}

The nuclear receptor REV-ERB $\alpha$ is part of the molecular clock mechanism and is considered to be involved in a variety of biological processes within metabolically active peripheral tissues as well. To investigate whether Rev-erb $\alpha$ (also known as Nr1d1) in the brain plays a role in the daily variations of energy metabolism, feeding behaviour and the sleep-wake cycle, we studied mice with global (GKO) or brain (BKO) deletion of Rev-erb $\alpha$. Mice were studied both in a light/dark cycle and in constant darkness, and then 24-hour variations of Respiratory quotient (RQ) and energy expenditure, as well as the temporal patterns of rest-activity and feeding behaviour, were recorded. The RQ increase of GKO mice was not detected in BKO animals, indicating a peripheral origin for this metabolic alteration. Arrhythmic patterns of locomotor activity were only found in BKO mice. By contrast, the circadian rhythm of food intake was lost both in GKO and BKO mice, mostly by increasing the number of daytime meals. These changes in the circadian pattern of feeding behaviour were, to some extent, correlated with a loss of rhythmicity of hypothalamic Hort (also named Orx) mRNA levels. Taken together, these findings highlight that Rev-erb $\alpha$ in the brain is involved in the temporal partitioning of feeding and sleep, whereas its effects on energy metabolism are mainly exerted through its peripheral expression.

\section{KEYWORDS}

circadian rhythm, clock gene, energy expenditure, feeding behaviour, hypothalamus, Nr1d1

\section{1 | INTRODUCTION}

Daily variations of behavioural and metabolic processes, such as the sleep-wake cycle and feeding-fasting rhythm, are controlled by a network of circadian clocks in the brain and peripheral tissues. In mammals, the main circadian clock is located in the suprachiasmatic nuclei of the hypothalamus, and is mostly reset by light perceived by the retina. ${ }^{1,2}$ Secondary clocks present in other cerebral regions and peripheral organs (eg, liver, muscle and pancreas) are adjusted in phase by the suprachiasmatic clock via the autonomic nervous system and endocrine signals. ${ }^{3,4}$ The secondary clocks can also be shifted by behavioural factors, such as food intake. ${ }^{5,6}$ The molecular clockwork is based on autoregulatory transcriptional/translational feedback loops that generate rhythmic transcriptional activity with an approximately 24 -hour period. In this network, two transcriptional activators, CLOCK and BMAL1, stimulate the expression of Period (Per1-3) and Cryptochrome (Cry1,2) genes, whose proteins in turn can repress the CLOCK-BMAL1 transactivation. ${ }^{7}$ In addition to these main components, the nuclear receptors $\operatorname{Ror}(\alpha, \beta, \gamma)$ and $\operatorname{Rev}$-erb $(\alpha, \beta)$ compete to activate and repress, respectively, the transcription of Bmal1 and Clock, thereby reinforcing the robustness of circadian oscillations. ${ }^{8-10}$ The amplitude of the circadian oscillations is further enhanced by targeted degradation of REV-ERB $\alpha .{ }^{11}$ 
Besides its role in the internal timing system, REV-ERB $\alpha$ is also involved in various cellular processes related to lipid metabolism, ${ }^{12,13}$ vascular inflammation ${ }^{14,15}$ and haem binding. ${ }^{16,17}$ Together, these results suggest that REV-ERB $\alpha$ is an intracellular integrator of metabolic and circadian pathways. ${ }^{18}$ Mice bearing a global deletion of Rev-erb $\alpha$ display an altered daily balance in fuel partitioning, leading to mistimed alternation between lipogenesis during the active/feeding period and lipolysis during the resting/fasting period. ${ }^{19}$ However, it is still unclear whether these changes associated with lack of Rev-erb $\alpha$ have a peripheral or central origin.

Feeding behaviour is strongly organised in time at both ultradian (ie, meal) and circadian scales (ie, daily feeding/fasting cycle). ${ }^{20}$ The regulation of energy balance is based on activation/repression of complex brain circuits controlling energy intake and expenditure. The main components of this neural network include the mediobasal hypothalamus (eg, arcuate and ventromedial nuclei) and the brainstem (eg, nucleus of the solitary tract and parabrachial nucleus). ${ }^{21,22}$ These structures are sensitive to circulating metabolic signals from peripheral tissues, such as glucose, non-esterified fatty acids and metabolic hormones (eg, leptin, insulin and ghrelin) and afferent inputs from the peripheral nervous system.

The coupling between the homeostatic regulation of energy metabolism and circadian clocks is not fully understood, although it is considered to involve cellular integrators of metabolic and circadian processes, such as REV-ERB $\alpha$. Daily changes in food availability elicit rhythmic behavioural, physiological and hormonal changes in anticipation of food access. ${ }^{6,23}$ Recent data indicate that brain expression of Rev-erb $\alpha$ largely contributes to the cerebral processes predicting food access because a brain deletion of Rev-erb $\alpha$ prevents these behavioural and thermogenic responses, whereas a global deletion of Rev-erb $\alpha$ only reduces food-anticipatory behaviour and thermogenesis. ${ }^{24}$ The daily rhythm of food intake and anticipation of food access may share neuronal substrates and functions, raising the possibility that brain REV-ERB $\alpha$ also participates in the temporal organisation of the daily feeding pattern.

To test whether Rev-erb $\alpha$ expressed in the brain indeed plays a role in the daily variations of energy metabolism and the temporal structure of feeding behaviour, we measured the respiratory quotient, locomotor activity and energy expenditure over 24 hours, and also determined the quantity and organisation of spontaneous food intake in mice with either a global or central deletion of Rev-erb $\alpha$ in a light/dark cycle. To establish whether these rhythms are driven by circadian clocks, they were also studied in constant darkness. To further understand the neural substrate underlying the behavioural changes observed quantitative polymerase chain reactions (PCRs) were performed on several hypothalamic tissues to study clock and metabolic genes.

\section{2 | MATERIALS AND METHODS}

\subsection{Animals and housing conditions}

The founder heterozygous mice bearing a global deletion of Rev-erb $\alpha$ (GKO) were kindly provided by Professor Ueli Schibler (University of Geneva, Geneva, Switzerland) and rederived on a C57BL6J background. The description of the Rev-erb $\alpha$ deletion is provided elsewhere. ${ }^{8}$ Controls of GKO mice were wild-type (WT) littermates. The conditional Rev-erb $\alpha$ knockout (KO) mice were generated at the Mouse Clinical Institute (Strasbourg, France) in the framework of the European EUMODIC consortium. ${ }^{25}$ Details for the genesis of this mouse line are provided elsewhere. ${ }^{24}$ To generate brain-specific KO mice, conditional Rev-erb $\alpha$ KO individuals were crossed with Nestin ${ }^{\text {Cre }}$ transgenic mice (line \#003771; Jackson Laboratories, Bar Harbor, ME, USA). To improve the efficiency of brain deletion of Rev-erb $\alpha$, we generated Reverbo ${ }^{f / g k o}$;Nes-Cre mice, hereafter designated as BKO mice, which carry one floxed allele ( $f l)$ and one global deleted ( $g k o$ ) allele, so that one Rev-erb $\alpha$ allele is deleted in every cell and, in addition, nervous cells do not express Rev-erb $\alpha$ because of Nestin-Cre inactivation of the other Rev-erb $\alpha$ allele. ${ }^{24}$ Controls of BKO mice were Rev-erb $\alpha^{\mathrm{fl} /+} ; \mathrm{Nestin}{ }^{\mathrm{Cre}}$ littermates (noted CTRL below), which carry one floxed allele (fl) and Cre recombinase transgene under the control of the Nestin promoter, thus impairing the expression of only one Rev-erb $\alpha$ allele in nervous cells.

Mice were bred within a pathogen-free animal care facility (Chronobiotron platform, UMS 3415, CNRS and University of Strasbourg) in a 12:12-hour light/dark cycle, with lights on (07.00 hours) defining Zeitgeber time (ZT) 0 and lights off (19.00 hours) defining ZT12, at $22 \pm 1^{\circ} \mathrm{C}$ and $55 \pm 5 \%$ humidity. Food (Rodent chow, SAFE diet 105; SAFE Augy, France; distribution of metabolisable energy content as percentage: $27 \%$ protein, $59 \%$ carbohydrate and $14 \%$ fat) and ultraviolet-treated tap water were provided ad lib. Two- to 4-month-old male mice were individually housed in transparent plastic cages enriched with nest cotton and a piece of wood. Body mass was measured before and after the indirect calorimetry experiment. Body size was measured from snout to beginning of tail. All experiments were performed in accordance with the NIH Guide for the Care and Use of Laboratory Animals (1996), the French National Law (implementing the European Union Directive 2010/63/EU) and were approved by the Regional Ethical Committee of Strasbourg for Animal Experimentation (CREMEAS) and the French Ministry of Higher Education and Research (APAFIS \#2533-2015110215078867 v1).

\subsection{Experimental design for indirect calorimetry}

Mice were kept individually in metabolic cages (floor area: $210 \mathrm{~cm}^{2}$ ) to measure daily patterns of energy expenditure (EE), respiratory quotient (RQ) using an open-circuit indirect calorimetry system (Addenfi, Les Cordeliers, France). Concentrations of $\mathrm{O}_{2}$ and $\mathrm{CO}_{2}$ in the outgoing air were successively measured in four different cages. Each cage was sampled every 15 minutes. The set-up also allowed feeding behaviour to be investigated using an automated weighing system, as well as locomotor activity using actimetry plates (ie, pressure sensors below the cage). In the metabolic cages, the same chow pellets and water were provided as in the home cage, except that food access was horizontal, instead of vertical, and water was made available in a cup, instead of a bottle. Mice were allowed to acclimatise to the metabolic cages during 3 consecutive days in LD conditions. Measurements were performed during days 4 and 5 , on day 4 in LD and on day 5 in constant darkness (DD). Body weight and food left over in the feeder was measured at 
the end of the experiment. Thereafter, mice were transferred back to their home cages in LD conditions for at least 2 weeks. Data from animals that lost more than $10 \%$ of initial body weight or that ate less than $3 \mathrm{~g} \mathrm{day}^{-1}$ in the metabolic cages were discarded. The final numbers of animals for analysis of calorimetry and feeding behaviour were: WT ( $n=8)$, GKO $(n=4), \operatorname{CTRL}(n=8)$ and BKO $(n=6)$.

\section{3 | Tissue and blood collection}

Groups of WT, GKO, CTRL and BKO mice were killed at ZT5, ZT11, ZT17 and ZT23 ( $n=4-5$ per group and ZT). Mice were injected i.p. with a lethal dose of pentobarbital $\left(200 \mathrm{mg} \mathrm{kg}^{-1}\right.$; CEVA, Libourne, France). An intracardial blood sample was taken with $4 \%$ ethylenediaminetetraacetic acid and brains were sampled and frozen in isopentane at $-35^{\circ} \mathrm{C}$. Plasma was sampled after blood centrifugation at $4600 \mathrm{~g}$ for 10 minutes at $4^{\circ} \mathrm{C}$. The hypothalamus was microdissected from the brain visually (ie, from the retrochiasmatic level to the hypothalamic posterior region, excluding the suprachiasmatic region harbouring the master clock). As noted elsewhere ${ }^{26}$ the rostrocaudal size of each sample extends from Bregma -0.9 to $-2.5 \mathrm{~mm}, \pm 1.5 \mathrm{~mm}$ on each side of the midline (ie, $3 \mathrm{~mm}$ width) and with a ventrodorsal height of $1.5 \mathrm{~mm}$ from the hypothalamic floor. The hypothalamic tissue block, which most likely includes all hypocretin neurones, was immediately frozen in liquid nitrogen and stored at $-80^{\circ} \mathrm{C}$ until the mRNA extraction procedure.

\section{4 mRNA extraction and quantitative real-time PCR of hypothalamic tissue}

Frozen hypothalamic tissue blocks from WT, GKO, CTRL and BKO were placed on ice and homogenised in lysis buffer supplemented with $\beta$-mercaptoethanol and total RNA was immediately extracted in accordance with the manufacturer's instructions (RNeasy Mini Kit; Qiagen, Valencia, CA, USA). RNA quantity and quality were measured using NanoDrop spectrophotometer (ND-1000; NanoDrop, Wilmington, DE, USA) and Bioanalyzer (Agilent RNA 6000 Pico kit; Agilent Technologies Inc., Santa Clara, CA, USA), respectively. cDNAs were synthesised from 200 ng of total RNA using the SuperScript III Kit (Invitrogen, Carlsbad, CA, USA). Quantitative real-time PCR was performed and analysed using a Real-time PCR System (7300; Applied Biosystems, Foster City, CA, USA) with 1X TaqMan Gene Expression Master Mix (Applied Biosystems), 1X TaqMan Gene Expression Assay (Applied Biosystems) and $1 \mu \mathrm{L}$ of cDNA in a total volume of $20 \mu \mathrm{L}$. PCR conditions consisted of 10 minutes at $95^{\circ} \mathrm{C}$ followed by 40 cycles of 15 seconds at $95^{\circ} \mathrm{C}$, and 1 minute at $60^{\circ} \mathrm{C}$. PCR reactions were performed in duplicate. Relative expression levels were determined using the comparative $\triangle \mathrm{CT}$ method. Expression levels of all genes of interest were standardised by normalisation over the geometric mean of two house-keeping genes: $\beta 2$-microglobulin and Hypoxanthine-guanine-phosphoribosyl-transferase (Hprt). The TaqMan Gene Expression Assays used were: $\beta 2$-microglobulin (Mm00500226_m1), Hprt (Mm00446968_m1), Rev-erba (also named Nr1d1, Mm00520711_g1), Bmal1 (Mm00500226_m1), Clock (Mm00455950_m1), Period 2 (Per2, Mm00478113_m1), Hypocretin (also named orexin, Hcrt, Mm01964030_s1), Neuropeptide Y (Npy, Mm03048253_m1), Pro-opiomelanocortin (Pomc, Mm00435874_m1) and Lipoprotein lipase (Lpl, Mm00434764_m1).

\section{5 | Plasma metabolic measurements}

Plasma glucose was evaluated with GOD-PAP Kit (Biolabo, Maizy, France). Concentrations of plasma non-esterified fatty acids (NEFA) were determined in accordance with the ACS-ACOD Method (NEFA-HR2; Wako, Osaka, Japan). Plasma insulin was assayed with an ultra-sensitive mouse insulin enzyme-linked immunoassay kit (ELISA) kit (CrystalChem, Downers Grove, IL, USA). Levels of plasma leptin were measured with a mouse leptin ELISA Kit (Crystal Chem).

\subsection{Analysis of feeding pattern and calorimetry}

For calorimetry analysis, both energy expenditure and RQ (ie, ratio of $\mathrm{CO}_{2}$ production over $\mathrm{O}_{2}$ consumption) were calculated using ALAB, version 1.55 (Addenfi, Paris, France). The same software was used to analyse the microstructure of feeding behaviour, including the amount of food consumed, number of eating bouts, number of meals and meal duration. Meals consisted of one or more eating bouts (ie, contacts with the feeder comprising a food intake of at least $0.05 \mathrm{~g}$ ) separated by an interprandial interval of at least 5 minutes. Thus, a new meal was considered to start if feeding bouts were $\geq 5$ minutes apart. These criteria are based on those reported previously, ${ }^{27}$ except for the minimal amount of dislodged food as a result of the lower precision of the Addenfi scale. Detection thresholds were $0.05 \mathrm{~g}$ for food intake, $5 \mathrm{~min}$ utes for inter-prandial intervals and $0.1 \mathrm{~g} \mathrm{~s}^{-1}$ for derived detection.

\section{7 | Statistical analysis}

All values are expressed as the mean \pm SEM. Statistical analysis was performed using SIGMAPLOT, version 12 (Systat Software Inc., San Jose, CA, USA).

Data were analysed with one- or two-way ANOVA followed by Fisher's least significant difference method and Tukey's honestly significant difference post-hoc analysis, respectively, when applicable. $P \leq .05$ was considered statistically significat. For assessing daily characteristics of feeding, locomotor activity and RQ, we used a cosinor analysis to determine mean level, amplitude and acrophase of each rhythm with SIGMAPLOT. Data were fitted to the regression: $y=a+b \cdot \cos (2 \pi[x-c] / 24)$, where $a$ is the mean level, $b$ is the amplitude, and $c$ is the acrophase of the rhythm. Cosinor regressions for the three fitted parameters were considered significant only when $P \leq .05$.

\section{3 | RESULTS}

\section{1 | Temporal feeding pattern in WT, GKO, CTRL and BKO mice}

Neither body mass, nor daily food intake differed significantly between WT, GKO, control and BKO mice fed ad lib. (Table 1) in 
agreement with previous studies. ${ }^{19,24}$ The lower body size in mice expressing Nestin ${ }^{\mathrm{Cre}}$ is also in accordance with other studies. ${ }^{28}$ There was a trend for increased adiposity in both GKO and BKO mice compared to the respective controls (Table 1). The daily pattern of food intake (higher at night) was similar, with no significant difference in the four genotypes housed in LD conditions, with an acrophase around ZT15. By contrast, after transfer to DD, GKO mice displayed increased food intake during subjective daytime and both GKO and BKO mice lost their circadian rhythm of food intake (Figure 1A,B). Regarding the microstructure of feeding behaviour, the daily rhythm in the number of meals (higher at night) did not differ according to the genotype in LD conditions (see Supporting information, Table S1). The peak in the number of daily meals in DD was delayed in BKO compared to CTRL mice but not in GKO compared to WT mice (see Supporting information, Table S2) Meal duration increased at night (peak before midnight) in all genotypes in LD conditions. Both GKO and BKO mice in DD displayed a shift in the daily peak of meal duration (ie, approximately 2.8 hours of delay in GKO and 4 hours of delay in BKO, respectively) compared to their control group (Figure 1E,F; see also Supporting information, Table S2). The number of eating bouts showed a daily rhythm in the 4 genotypes in LD conditions. After transfer to DD, this rhythm was not markedly changed in GKO mice, although it disappeared in BKO mice (Figure 1G,H; see Supporting information, Table S2).

\section{2 | Daily variations of energy metabolism}

The daily pattern of locomotor activity was very similar between WT and GKO mice, except that its amplitude was decreased in GKO in LD conditions but not in DD. By contrast, BKO mice showed an arrhythmic profile of rest-activity, whereas CTRL mice displayed a dampened activity pattern, confirming previous observations (Figure 2A,B; see Supporting information, Table S3). ${ }^{24}$ Mean RQ, whose decreased and increased values reflect, respectively, higher utilisation of lipids and carbohydrates, was higher in GKO compared to WT mice in LD conditions but not in DD. The amplitude of $\mathrm{RQ}$ in LD conditions was dampened in BKO compared to CTRL animals, whereas mean values in DD were increased in BKO mice (Figure 2C,D). Daily variations of energy expenditure were comparable between WT and GKO mice in both LD and DD. By contrast, mean energy expenditure over 24 hours and the amplitude of the EE rhythm was consistently reduced in BKO compared to CTRL mice in both LD and DD (Figure 2E,F).

\section{3 | mRNA expression of clock and metabolic genes in brain hypothalamic punches}

To evaluate the role of Rev-erb $\alpha$ on gene expression in the metabolic hypothalamus, a hypothalamic tissue block was taken excluding the suprachiasmatic nuclei to limit phase-dispersion between the master clock and secondary hypothalamic clocks.

Daily expression of Rev-erb $\alpha$ was rhythmic with a peak in late daytime in the hypothalamus of both WT and CTR mice, whereas it was undetectable or at very low levels in GKO and BKO mice, respectively (Figure 3A). As expected by the lack of repressive effects of Rev-erb $\alpha$ on Bmal1 transcription, daily levels of Bmal1 expression were up-regulated in both GKO and BKO animals $(P<.05)$ compared to respective controls (WT and CTRL). Rhythmic expression of Bmal1 in WT mice peaked in late night (ie, ZT22), while Bmal1 expression showed no daily rhythmicity in the other 3 genotypes (namely, GKO, CTRL and BKO) indicating, in the case of CTRL, that the presence of only one Rev-erb $\alpha$ allele in cells was not sufficient to maintain a rhythmic expression of Bmal1. Daily expression of Clock was rhythmic in WT mice with a peak late night (ie, ZT21), in phase with that of Bmal1. By contrast, Clock expression lost its rhythmicity in both GKO and BKO mice and tended to show higher daytime values (statistically nonsignificant). Clock expression was rhythmic in CTRL and WT, although the mean levels of BKO were significantly different from WT and GKO (Table 2). Unexpectedly, a significant rhythmic expression of Per2 was detected in all genotypes without significant changes in the acrophase or amplitude (Figure 3D and Tables 2; see Supporting information, Table S4). We interpret the rhythmic expression of Per2 as being induced by rhythmic cues from brain clocks outside the sampled tissue block (eg, suprachiasmatic nuclei) and/or blood. This possibility is

TAB LE 1 Mean value data for the food intake, body weight, white adipose tissue (WAT), body size and WAT/body weight, comparison between all four genotype.

\begin{tabular}{llllll} 
Genotype & $\begin{array}{l}\text { Food intake }(\mathrm{g}) \\
\text { Mean } \pm \text { SEM }\end{array}$ & $\begin{array}{l}\text { Body weight }(\mathrm{g}) \\
\text { Mean } \pm \text { SEM }\end{array}$ & $\begin{array}{l}\text { WAT }(\mathrm{g}) \\
\text { Mean } \pm \text { SEM }\end{array}$ & $\begin{array}{l}\text { Body size }(\mathrm{mm}) \\
\text { Mean } \pm \text { SEM }\end{array}$ & $\begin{array}{l}\text { WAT/body weight } \\
\text { Mean } \pm \text { SEM }\end{array}$ \\
WT & $4.15 \pm 0.41$ & $34.69 \pm 0.95^{\mathrm{a}}$ & $1.09 \pm 0.15$ & $100.37 \pm 0.98^{\mathrm{a}, \mathrm{b}}$ & $0.031 \pm 0.03$ \\
GKO & $4.18 \pm 0.38$ & $33.62 \pm 3.85^{\mathrm{c}, \mathrm{d}}$ & $1.60 \pm 0.54$ & $100.75 \pm 2.01^{\mathrm{c}, \mathrm{d}}$ & $0.040 \pm 0.01$ \\
CTRL & $4.20 \pm 0.41$ & $29.85 \pm 0.72$ & $1.11 \pm 0.03$ & $92.85 \pm 1.51$ & $0.037 \pm 0.01$ \\
BKO & $3.89 \pm 0.14$ & $31.25 \pm 1.43$ & $1.46 \pm 0.23$ & $94.50 \pm 1.20$ & $0.046 \pm 0.06$ \\
\hline
\end{tabular}

One-way ANOVA comparisons of each parameter (ie, food intake, body weight, WAT, body size and WAT/body weight ratio) between wild-type (WT) mice; mice bearing a global deletion of Rev-erb $\alpha$ (GKO); Control (CTRL) mice; and mice with brain deletion of Rev-erb $\alpha$ (BKO).

aWT is different from control.

${ }^{b} \mathrm{WT}$ is different from BKO.

${ }^{\mathrm{C}} \mathrm{GKO}$ is different from control.

${ }^{\mathrm{d} G K O}$ is different from BKO. 

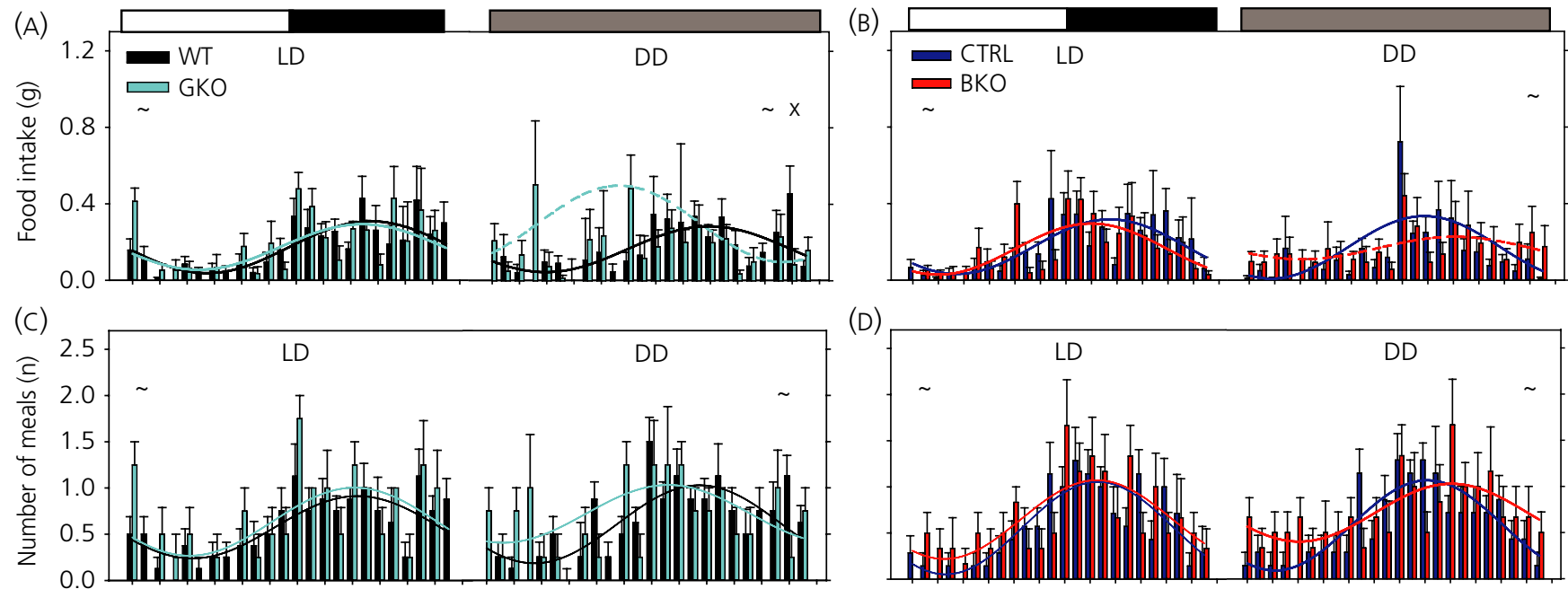

(E)

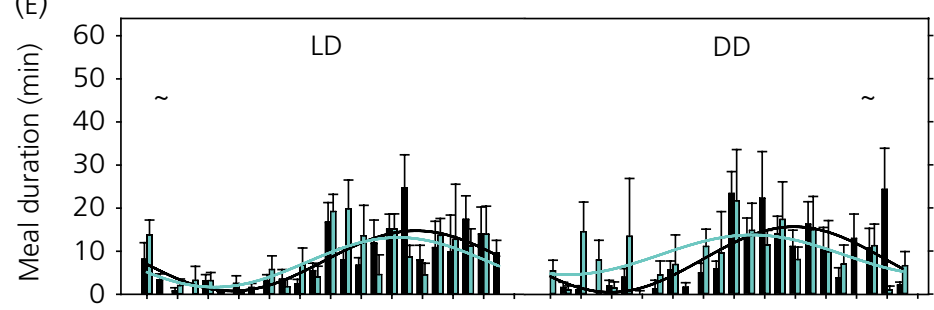

(F)

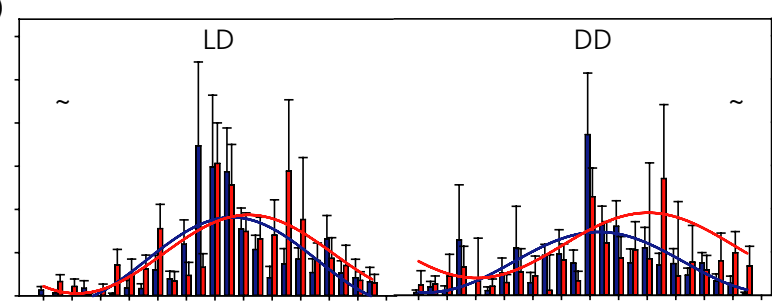

$(\mathrm{G})$

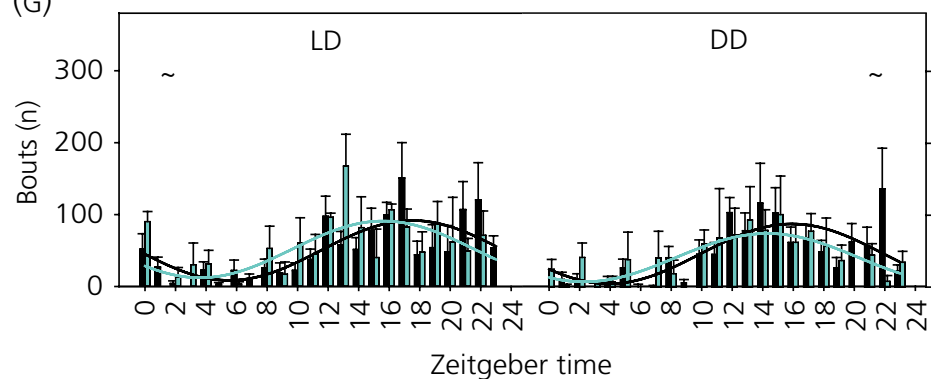

(H)

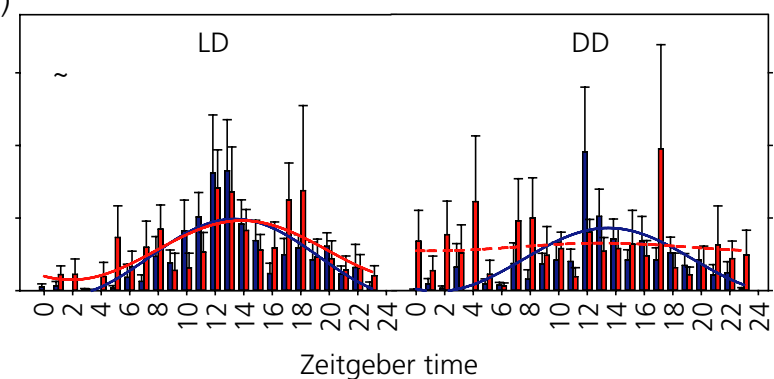

FIG URE 1 Daily profiles of feeding parameters in mice in light/dark (LD) conditions and constant darkness (DD). Food intake (A, B), number of meals (C, D), meal duration (E, F) and bouts (G, H) in wild-type (WT) mice; mice bearing a global deletion of Rev-erb $\alpha$ (GKO); Control (CTRL) mice; and mice with brain deletion of Rev-erb $\alpha$ (BKO). Black bars represent WT mice; cyan bars represent GKO mice; blue bars represent CTRL mice; and red bars represent BKO mice. Fitted solid and dashed lines represent significant and nonsignificant cosinor regressions, respectively. White and black rectangles on the $x$-axis represent 12:12-hour LD cycles and dark grey rectangles on the $x$-axis represent subjective day and subjective night in DD. , Effect of time; $x$, interaction between genotype and time $(P<0.05)$

supported by the fact that, when the liver clock is genetically arrested, hepatic expression of Per2 is still rhythmic as a result of rhythmic systemic signals. $^{29}$

Next, we evaluated the hypothalamic levels of genes coding for orexigenic or anorexigenic peptides. Expression of Hcrt was rhythmic in both control groups (WT and CTRL), although the mean levels were down-regulated in CTRL animals. Rhythmicity of Hcrt was lost in both GKO and BKO and expression levels were kept at intermediate values compared to their respective control groups. The expression of both Npy and Pomc was not rhythmic in any genotype. Mean levels of expression of both Npy and Pomc were higher in WT and GKO compared to CTRL and BKO mice.

Finally, we investigated the hypothalamic levels of $L p l$ which were found to be constitutively expressed in all genotypes, except in GKO mice, which displayed a small-amplitude rhythm with a low mean level of expression (Table 2). Therefore, the up-regulated levels of $L p l$ mRNA previously observed in the liver, white adipose tissue and skeletal muscle of GKO mice are likely specific to peripheral tissues. ${ }^{19}$

\subsection{Daily pattern in plasma metabolites and hormones}

The daily pattern of plasma glucose in WT mice, albeit not significantly rhythmic, was comparable to that previously described for ad lib. fed normal mice. ${ }^{30,31}$ Daily levels of plasma glucose in GKO mice were not significantly different from the WT mice, although a trend for increased glyceamia was visible in the late night, whereas 24-hour glycaemia was 
(A)

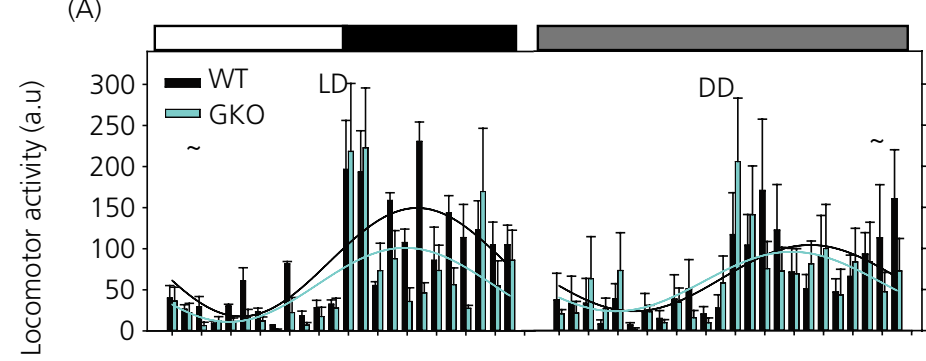

(C)

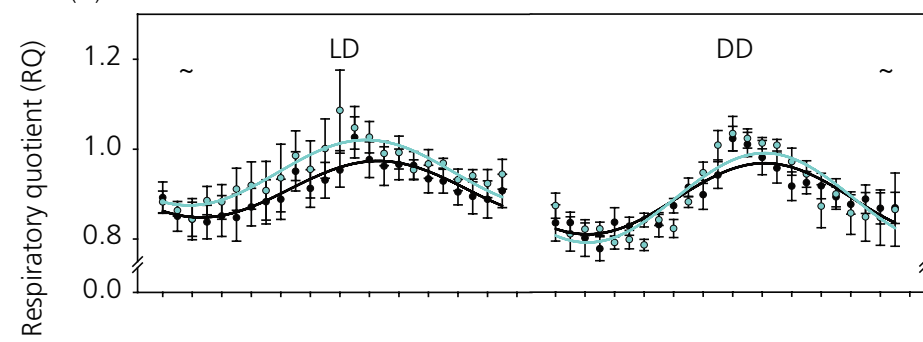

(B)

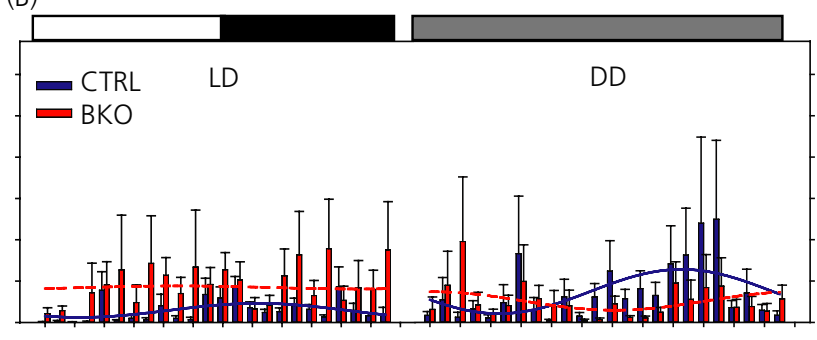

(D)

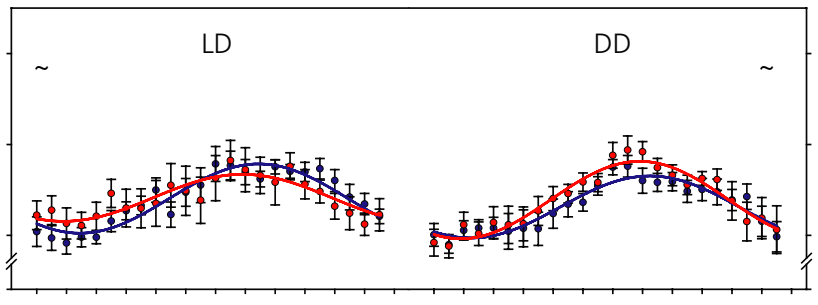

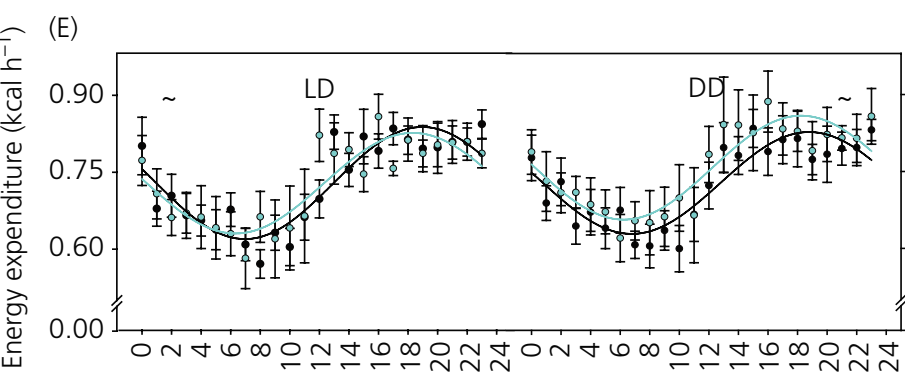

(F)

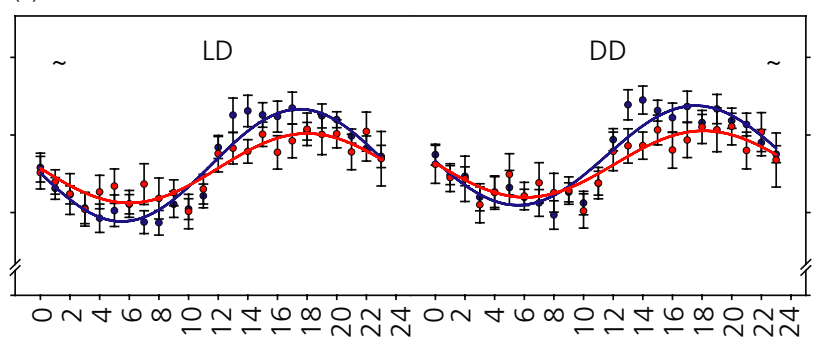

FIGURE 2 Daily profiles of physiological parameters in mice in light/dark (LD) conditions and constant darkness (DD). Locomotor activity $(A, B)$, respiratory quotient (C, D) and energy expenditure (E, F). Black bars represent wild-type (WT) mice; cyan bars represent mice bearing a global deletion of Rev-erb $\alpha$ (GKO); blue bars represent Control (CTRL) mice; and red bars represent mice with brain deletion of Rev-erb $\alpha$ (BKO). Fitted solid and dashed lines represent significant and nonsignificant cosinor regressions, respectively. White and black rectangles on the $x$-axis represent 12:12-hour LD cycles and dark grey rectangles on the $x$-axis represent subjective day and subjective night in DD. , Effect of time $(P<.05) . \mathrm{AU}$, arbitrary unit

similarly low during late daytime and early night in both CTRL and BKO mice (Figure 4A; see also Supporting information, Table S4). A difference in baseline glycemia between Nestin-Cre (CTRL) and mice floxed for another gene has not been reported previously. ${ }^{32}$

Plasma levels of NEFA were constant across the day in WT, GKO and $\mathrm{BKO}$ mice and rhythmic with a small amplitude in CTRL animals (Figure 4B).

Plasma insulin in WT mice showed a significant daily rhythm with increased levels at night, as found in other mouse studies. ${ }^{30,33}$ In the other 3 genotypes, no significant daily variations were detected for plasma insulin and a two-way ANOVA indicates no significant effect of genotype and interaction ( $P=.17$ and $P=.42$, respectively), although it did detect an effect of Time $(P=.005)$ (see Supporting information, Table S4). A previous study ${ }^{32}$ suggested that a defect in insulin secretion in Nestin-Cre (CTRL) mice may explain the trend for lower insulinaemia at night.

Daily levels of plasma leptin were also rhythmic in WT, with a peak around midnight as described previously, ${ }^{30}$ whereas no significant rhythms were observed in the other 3 genotypes (Figure 4D). A higher daytime leptinemia in Nestin-Cre (CTRL) mice as compared to mice floxed for another gene has also been described previously. ${ }^{32}$

\section{4 | DISCUSSION}

The transcriptional repressor REV-ERB $\alpha$ has been implicated in the molecular basis of circadian clocks and in an array of biological processes within metabolically active peripheral tissues. ${ }^{34}$ The present study shows that brain Rev-erb $\alpha$ participates in the control of the circadian rhythm in feeding behaviour, as indicated by the loss of a daily food intake rhythm both in mice lacking Rev-erb $\alpha$ in all cells (GKO) or specifically in neurones (BKO). Loss of the feeding rhythm was detected in constant darkness but not in a light/dark cycle, demonstrating that constant dark unmasks the inhibitory effect of daytime light on this behaviour. The implication of brain Rev-erbo in the sleep/ wake activity rhythm was confirmed by the arrhythmic patterns of locomotor activity in BKO mice both in a light/dark cycle and in constant darkness. The arrhythmic behaviour of the BKO animals was also reflected in the reduced amplitude of the rhythms in EE. By 
FIGURE 3 Daily expression of clock, orexigenic, anorexigenic and metabolic genes in the hypothalamus of wild-type (WT) mice; mice bearing a global deletion of Rev-erb $\alpha$ (GKO); Control (CTRL) mice; and mice with brain deletion of Rev$e r b \alpha$ (BKO) in light/dark (LD) conditions. Expression of clock genes Rev-erb $\alpha$ (A), Bmal1 (B), Clock (C) and Per2 (D); orexigenic genes Npy (E) and Hcrt (G); anorexigenic gene Pomc (F); and metabolic gene $L p l(H)$. Black circles represent WT mice; a cyan rhombus represents GKO mice; a blue square represents $\mathrm{CTRL}$ ) mice; and a red triangle represents BKO mice. $X$, interaction between genotype and time; , effect of time; \#, effect of genotype $(P<.05)$. Fitted solid and dashed lines represent significant and nonsignificant cosinor regressions, respectively. $\mathrm{AU}$, arbitrary unit
(A)

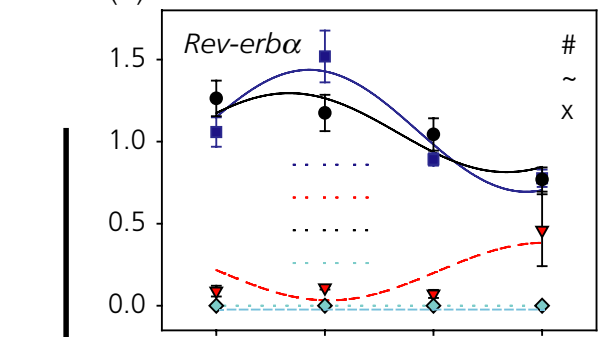

(c)

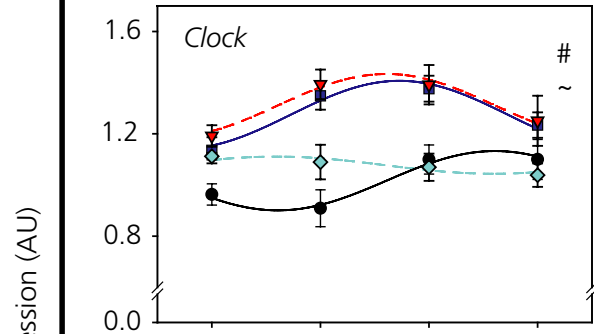

(E)

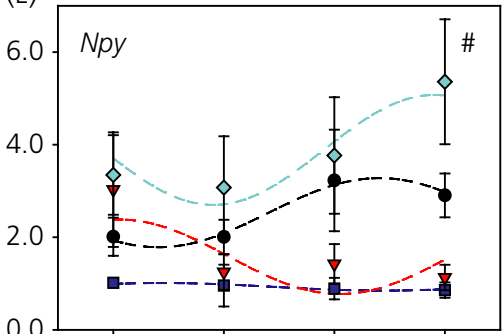

(G)

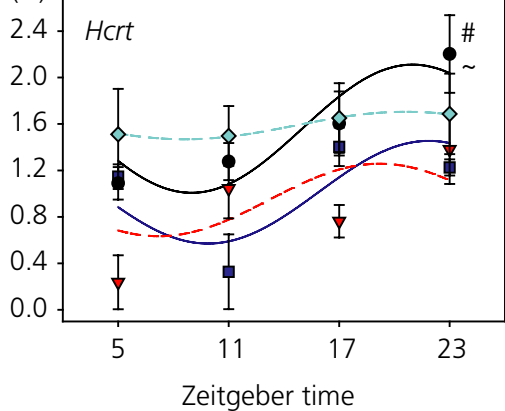

(B)

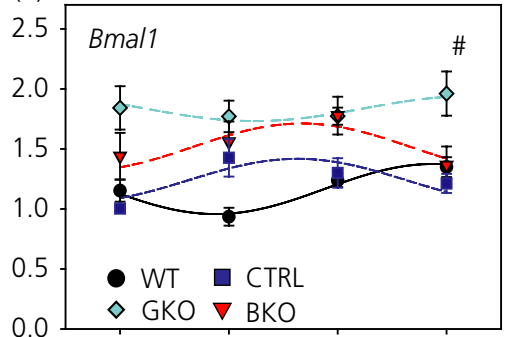

(D)

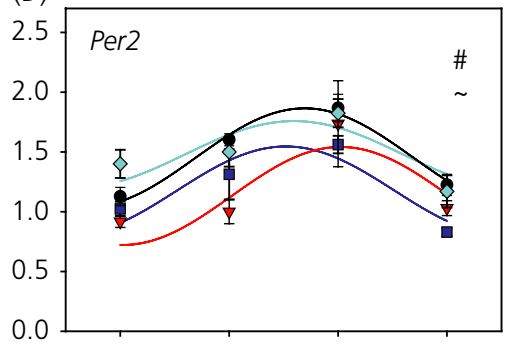

(F)

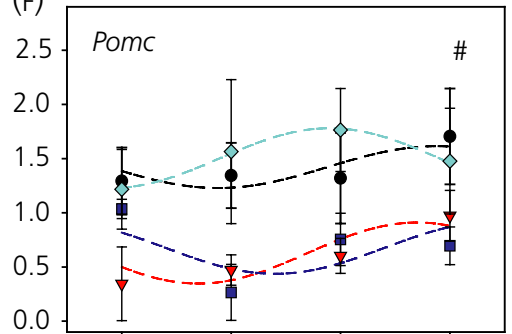

(H)

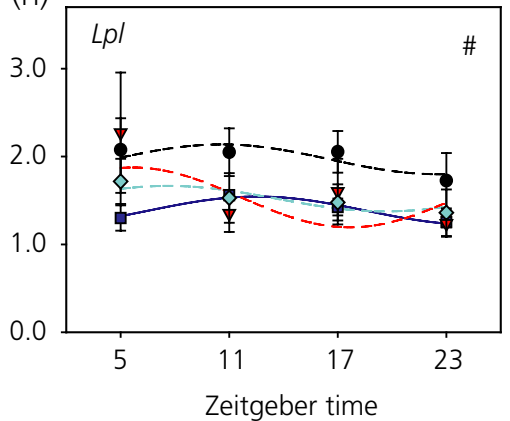

contrast, the increase of RQ in GKO mice was not detected in BKO animals, indicative of a peripheral origin for this metabolic change.

\section{1 | Daily metabolism}

Daily variations of energy expenditure were very similar in Rev-erb $\alpha$ GKO and WT mice, although they were reduced in Rev-erb $\alpha$ BKO compared to control mice. The latter most likely is related to the arrhythmic pattern of rest-activity in mice with brain deletion of Rev$e r b \alpha$. The lack of a clear correlation between the locomotor activity rhythm and energy expenditure may be a result of the high sensitivity of the pressure sensors below the cage that were used to detect motor activity. In other words, small movements (eg, for grooming) will not markedly increase energy expenditure but will be recorded by the pressure sensors as movements. In terms of rhythmicity, the maintenance of a daily rhythm of RQ in GKO and BKO mice in both
LD and DD suggests either that brain REV-ERB $\alpha$ is not critical, or that REV-ERB $\beta$ compensates for brain control of RQ rhythm (see below).

GKO mice showed higher rhythmic RQ values than those in WT mice, especially in LD conditions. This increase reflects a globally lower reliance on fat utilisation as an energy source. Previous data indicate that higher RQ values at night in GKO mice may be caused by de novo lipogenesis from dietary carbohydrates. ${ }^{19} \mathrm{~A}$ similar effect was not visible in BKO animals, therefore suggesting its peripheral origin. In other words, the RQ change in GKO mice would result from the lack of Rev-erb $\alpha$ in peripheral tissues. Findings comparable to those in GKO mice have been obtained in mice with a global deletion of Bmal1. ${ }^{35}$ Given that REV-ERB $\alpha$ is a circadian repressor of Bmal1 transcription, it is surprising that both genotypes lead to analogous metabolic effects, unless they result from an altered clockwork output rather than deletion of a specific circadian gene. The fact that the difference in RQ disappeared in GKO mice transferred to constant darkness suggests 
TAB LE 2 Cosinor parameters for clock, orexigenic, anorexigenic and metabolic genes in the hypothalamus

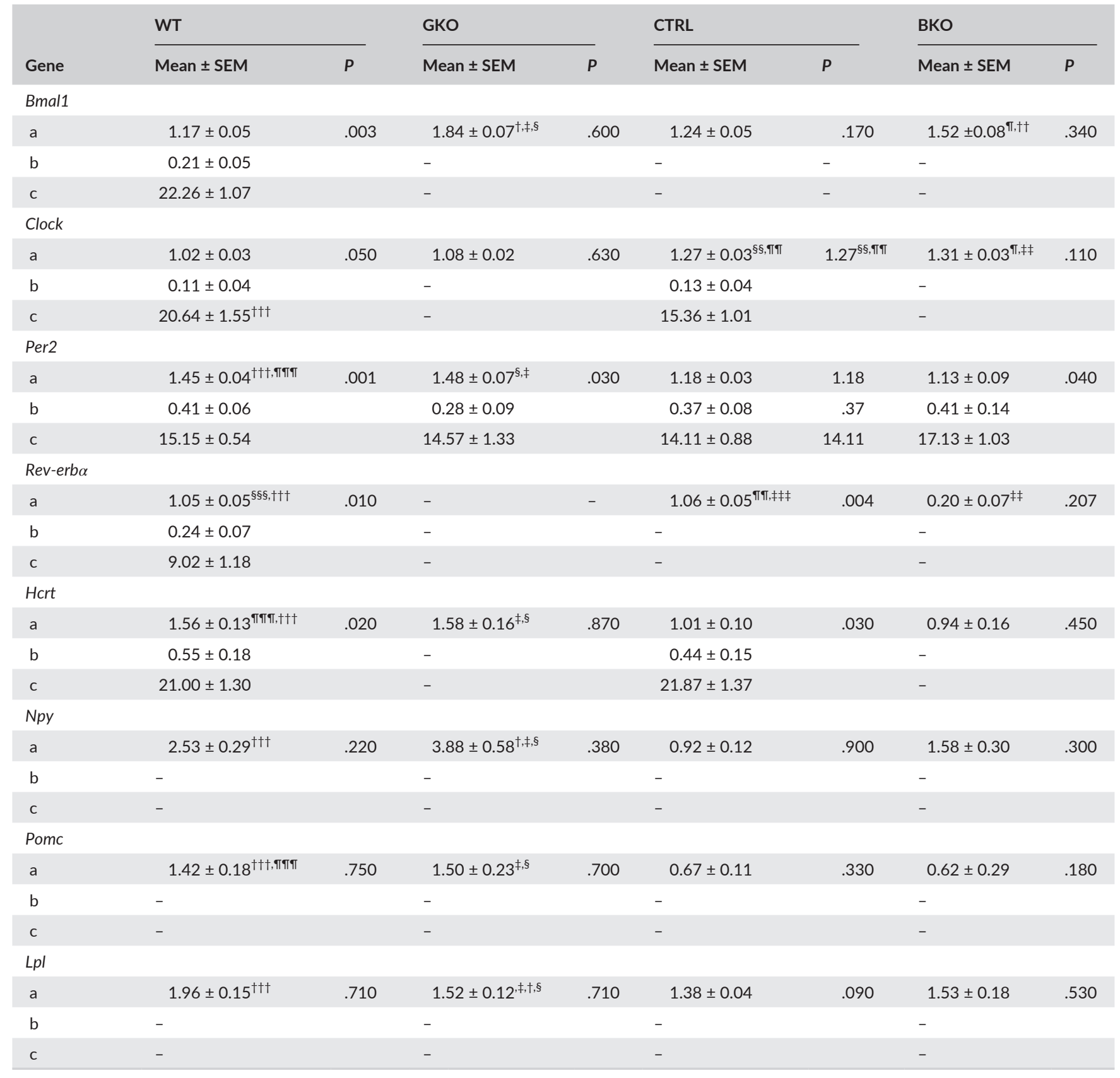

The three fitted parameters of cosinor regressions, including "a" (the mean level); "b" (the Amplitude); and "c" (the acrophase of the rhythm; for details, see Materials and methods). For the acrophase, the reference time is Zeitgeber 0 (ie, lights on). WT, wild-type mice; GKO, mice bearing a global deletion of Rev-erb $\alpha$; CTRL, Control mice; BKO, mice with brain deletion of Rev-erb $\alpha$.

$P$-values on the right column indicate significance of the cosinor analysis. Nonsignificant parameters are not shown (-).

${ }^{\dagger} \mathrm{GKO}$ is different from WT $(P<.05)$.

${ }^{\ddagger} \mathrm{GKO}$ is different from Control.

${ }^{\S} \mathrm{GKO}$ is different from BKO.

"BKO is different from WT.

${ }^{\dagger} \mathrm{BKO}$ is different from Control.

${ }^{\ddagger \ddagger} \mathrm{BKO}$ is different from GKO.

${ }^{\S \S}$ Control is different from WT.

आब Control is different from GKO.

${ }^{+t \dagger} \mathrm{WT}$ is different from Control.

${ }^{\ddagger \ddagger}$ Control is different from BKO.

${ }^{\S \S} W \mathrm{t}$ is different from GKO.

आศा $W \mathrm{t}$ is different from BKO $(P<.05)$. 
FIGURE 4 Daily profiles of plasma metabolites in wild-type (WT) mice; mice bearing a global deletion of Rev-erb $\alpha$ (GKO); Control (CTRL) mice; and mice bearing a global deletion of Rev-erb $\alpha$ (GKO) mice in light/dark (LD) conditions. Plasma glucose (A), plasma non-esterified fatty acids (NEFA) (B), plasma insulin (C) and plasma leptin (D). Black circle represents WT mice; a cyan rhombus represents GKO mice; a blue square represents CTRL mice; and a red triangle represent $\mathrm{BKO}$ mice. Fitted solid and dashed lines represent significant and nonsignificant cosinor regressions, respectively. White and black rectangles on the $x$-axis represent 12:12-hour LD cycles. \#, Effect of genotype; , effect of time $(P<.05)$
(A)

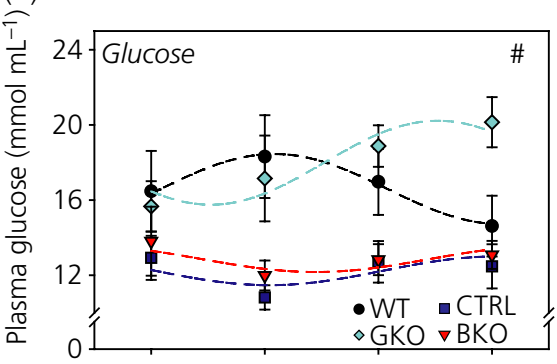

(C)

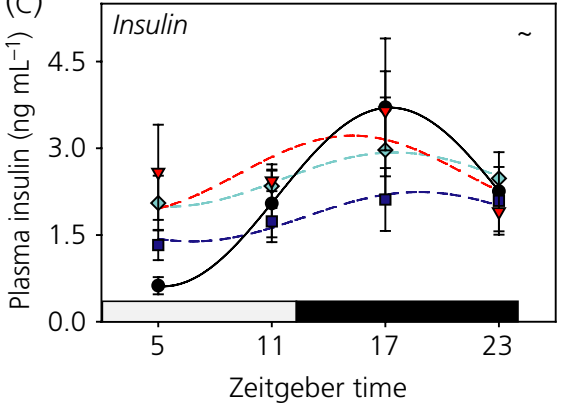

(B)

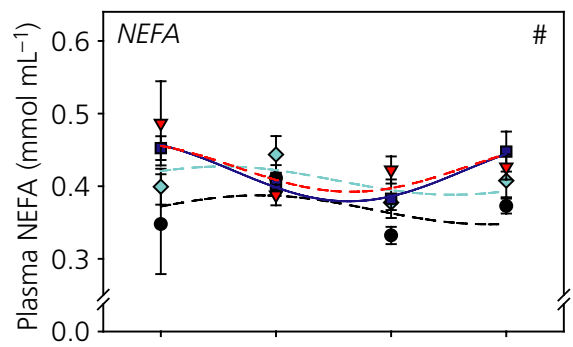

(D)

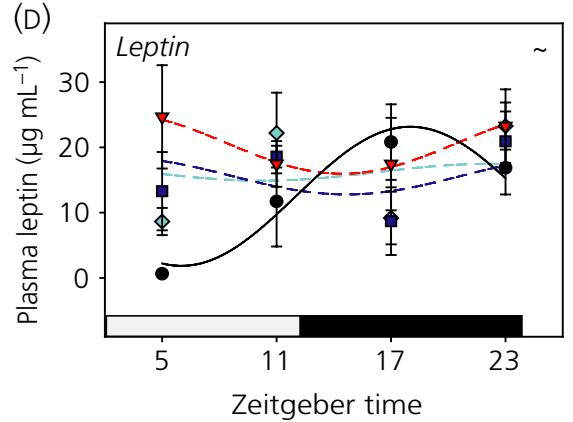

that light during light/dark cycles exerts direct effects on this outcome. How light would increase RQ during the resting period of GKO mice in light/dark cycles is not clear. Changes in feeding behaviour are unlikely to be involved because the lower food intake during the resting phase in light/dark cycles would rather decrease RQ values at that time. Alternatively, putative activation of the sympathetic nervous system by light would rather favour glucose oxidation, thus increasing $\mathrm{RQ}$ values.

\section{2 | Temporal pattern of rest-activity}

The circadian control of the rest-activity cycle relies on brain clocks, especially on the master clock in the suprachiasmatic nuclei. Rev-erb $\alpha$ GKO mice display mild alterations in the rest-activity rhythm, including a lower amplitude of the activity rhythm in a light/dark cycle (present study) and a shorter free-running period in constant darkness. ${ }^{8}$ GKO mice also showed altered sleep homeostasis in a light/dark cycle, as indicated by a slower increase of the homeostatic need of sleep during wakefulness. ${ }^{36}$ By contrast, BKO mice expressed an arrhythmic pattern of rest-activity in LD and DD conditions, with their circadian behavioural phenotype being altered to a greater extent compared to GKO animals, confirming previous observations with another actimetry set-up. ${ }^{24}$ Previous studies have also noted dissociations between locomotor activity and metabolic rhythms in mice with impaired clocks. In Cry1/2 GKO mice in LD conditions, locomotor activity tends to be arrhythmic, whereas their daily RQ appears to be rhythmic. ${ }^{37}$ In the present study, this distinct effect is probably not a result of the differently mutated Rev-erb $\alpha$ and truncated protein products because, in both BKO and GKO, the deletion targets the DNA binding domain (ie, exons 3 and 4) of the Rev-erb $\alpha$ allele., ${ }^{8,24}$ REV-ERB $\beta$, a transcriptional repressor sharing many properties with REV-ERB $\alpha$, can compensate some circadian and metabolic functions affected by the knockdown of Rev-erb $\alpha$, as shown in the liver. ${ }^{38}$ Therefore, REV-ERB $\beta$ in GKO mice may have partly compensated for Rev-erb $\alpha$ deletion during early development. The late ontogenic expression of Nestin in the brain ${ }^{39}$ may have precluded compensatory mechanisms in brain structures controlling the rest-activity rhythm in BKO mice, thus explaining their stronger disturbance of the circadian phenotype. Furthermore, behavioural phenotyping of $\mathrm{Nestin}^{\mathrm{Cre}}$ mice revealed no changes in levels of locomotion and general exploratory activity during open-field tests, ${ }^{28}$ or general activity measured in cages with a wheel. ${ }^{24}$ Here, we noted a lower nocturnal activity in control Nestin ${ }^{\text {Cre }}$ mice compared to WT mice, possibly as a result of the different way that locomotion was detected in the present study (ie, pressure sensors instead of infrared beams). At present, it cannot be excluded that this behavioural response apparently linked to Nestin-Cre activity may somehow have exacerbated the alteration of rest-activity rhythm in BKO mice.

\section{3 | Temporal feeding pattern}

Normal amounts of food intake were consumed by mice with global or central deletion of Rev-erb $\alpha$ compared to their respective control littermates. Accordingly, we found no effect of i.c.v. injections of the REV-ERB $\alpha$ agonist (GSK4112) on the feeding behaviour of WT mice (S. Sen and E. Challet, unpublished data). A previous study also reported no change on total daily food intake in mice injected i.p. with REV-ERB $\alpha$ agonists. ${ }^{40}$ Together, these data indicate that Rev-erb $\alpha$ expression in the brain does not play any significant role in the homeostatic regulation of food intake.

By contrast, daily patterns of food intake were arrhythmic in Reverb $\alpha \mathrm{GKO}$ and $\mathrm{BKO}$ mice housed in $\mathrm{DD}$, highlighting the involvement of brain REV-ERB $\alpha$ in the circadian control of feeding behaviour. Such a behavioural impairment was not detected in LD conditions. There is a circadian clock in the mouse retina ${ }^{41}$ that may also participate in 
the observed changes. In our opinion, however, this is unlikely because, if the retinal clock was dramatically affected, major effects in behaviour and metabolism would be expected not only in DD, but also in LD conditions. In nocturnal rodents, environmental lighting directly modulates food intake, with light at night and a dark pulse during the daytime being anorexigenic and orexigenic, respectively. ${ }^{42} \mathrm{~A}$ plausible explanation in GKO and BKO mice is thus a masking effect of light during the regular light period in LD conditions that, every day, inhibits food intake during the daytime, overwhelming the drive for foraging during the resting period.

To date, other strains of mice bearing a mutated clock gene, such as Clock mutation and Staggerer (ie, mutated Ror $\alpha$ ), as well as those being GKO for clock genes, such as Per1/2 and Cry1/2 KO mice, also show an attenuated diurnal feeding rhythm, which arises from almost equal amounts of food eaten during daytime and night-time. ${ }^{43-46}$ The present data in Rev-erb $\alpha$ BKO further indicate that this behavioural deficit has a central origin that does not rely on impaired peripheral cues.

Meal pattern analysis is useful for understanding the mechanisms that control feeding behaviour. To our knowledge, the present study is the first to investigate the microstructure of meal patterns in mice KO for a clock gene. The microstructure data show that the increased food intake of Rev-erb $\alpha$ GKO mice during the subjective day is caused by both an increased number of meals and an increase of their duration, especially during the first half of the subjective light period. These changes could be related to the orexigenic effect of up-regulated Hcrt mRNA levels in the hypothalamus of GKO mice during the daytime. Such diurnal up-regulation fits with the presence of Retinoic acidrelated Orphan receptor Response Element (RORE) binding sites for Rev-Erbo in the promoter of the Hcrt gene. ${ }^{47}$ The arrhythmic pattern of Hcrt expression in both GKO and BKO mice exposed to a light/ dark cycle when their feeding pattern is still rhythmic suggests, again, that light during daytime may partly have inhibited any increase in eating during daytime (ie, light is masking the feeding behaviour but not hypothalamic neuropeptide expression). Daily expression of mRNA coding for other hypothalamic neuropeptides involved in food intake (ie, Npy and Pomc) did not display changes that could account for increased daytime food intake, except possibly the trend for enhanced expression of Npy mRNA in GKO mice during late night. The lack of daily fluctuations of Npy and Pomc expression in the hypothalamus has been already reported in rodents. ${ }^{48-50}$ In BKO mice, the disappearance of the circadian rhythm in food intake is mainly caused by an increased number of meals and eating bouts, during subjective daytime. Additional experiments are needed to investigate the circadian expression of hypothalamic neuropeptides in these mice exposed to constant darkness.

Together, these behavioural data suggest that the expression of Rev-erb $\alpha$ in the central nervous system is critically involved in the timing of the daily feeding rhythm, probably by affecting the hypothalamic orexin system, although not markedly in the integration of gut-derived satiation signals by the metabolic brainstem.

Further investigations will be needed to understand in more detail how and in which brain regions REV-ERB $\alpha$ modulates the circadian timing of feeding behaviour and the sleep/wake cycle.

\section{ACKNOWLEDGEMENTS}

We are indebted to Professor Ueli Schibler (University of Geneva, Switzerland) for kindly providing the founder Rev-erb ${ }^{+/-}$mice, as well as Professor Rüdiger Klein (Max Planck Institute of Neurobiology, München, Germany) for his donation of the Nestin-Cre line to Jackson Laboratories. We also thank the European EUMODIC consortium for generating the Rev-erb $\alpha$ floxed line, as well as the Institut Clinique de la Souris (ICS, Illkirch-Graffenstaden, France) for supplying pairs of these mice. This work was supported by doctoral fellowships from "Neurotime" Erasmus Mundus program, European Doctoral College of University of Strasbourg and Eurometropolis of Strasbourg (S.S.) and recurrent grants from the Centre National de la Recherche Scientifique, University of Strasbourg (E.C.), and University of Amsterdam (A.K.).

\section{CONFLICT OF INTERESTS}

The authors declare that they have no conflicts of interest.

\section{ORCID}

S. Sen (ID http://orcid.org/0000-0002-0950-9722

\section{REFERENCES}

1. Golombek DA, Rosenstein RE. Physiology of circadian entrainment. Physiol Rev. 2010;90:1063-1102.

2. Brown TM. Using light to tell the time of day: sensory coding in the mammalian circadian visual network. J Exp Biol. 2016;219: 1779-1792.

3. Buijs RM, Kalsbeek A. Hypothalamic integration of central and peripheral clocks. Nat Rev Neurosci. 2001;2:521-526.

4. Challet E. Keeping circadian time with hormones. Diabetes Obes Metab. 2015;17(suppl):176-183.

5. Schibler U, Ripperger J, Brown SA. Peripheral circadian oscillators in mammals: time and food. J Biol Rhythms. 2003;18:250-260.

6. Tahara Y, Shibata S. Chronobiology and nutrition. Neuroscience. 2013;253:78-88.

7. Ko $\mathrm{CH}$, Takahashi JS. Molecular components of the mammalian circadian clock. Hum Mol Genet. 2006;15 Spec No 2:R271-R277.

8. Preitner N, Damiola F, Lopez-Molina L, et al. The orphan nuclear receptor REV-ERBalpha controls circadian transcription within the positive limb of the mammalian circadian oscillator. Cell. 2002;110:251-260.

9. Guillaumond F, Dardente H, Giguere V, Cermakian N. Differential control of Bmal1 circadian transcription by REV-ERB and ROR nuclear receptors. J Biol Rhythms. 2005;20:391-403.

10. Crumbley C, Burris TP. Direct regulation of CLOCK expression by REV-ERB. PLoS One. 2011;6:e17290.

11. Zhao $X$, Hirota $T$, Han $X$, et al. Circadian amplitude regulation via FBXW7-targeted REV-ERBalpha degradation. Cell. 2016;165:1644-1657.

12. Zhang Y, Fang B, Damle M, et al. HNF6 and Rev-erbalpha integrate hepatic lipid metabolism by overlapping and distinct transcriptional mechanisms. Genes Dev. 2016;30:1636-1644.

13. Feng $D$, Liu $T$, Sun Z, et al. A circadian rhythm orchestrated by histone deacetylase 3 controls hepatic lipid metabolism. Science. 2011;331:1315-1319.

14. $\mathrm{Ma} \mathrm{H}$, Zhong $\mathrm{W}$, Jiang $\mathrm{Y}$, et al. Increased atherosclerotic lesions in LDL receptor deficient mice with hematopoietic nuclear receptor Reverbalpha knock-down. J Am Heart Assoc. 2013;2:e000235. 
15. Sato S, Sakurai T, Ogasawara J, et al. A circadian clock gene, Rev-erbalpha, modulates the inflammatory function of macrophages through the negative regulation of Ccl2 expression. J Immunol. 2014;192:407-417.

16. Raghuram S, Stayrook KR, Huang P, et al. Identification of heme as the ligand for the orphan nuclear receptors REV-ERBalpha and REVERBbeta. Nat Struct Mol Biol. 2007;14:1207-1213.

17. Yin L, Wu N, Curtin JC, et al. Rev-erbalpha, a heme sensor that coordinates metabolic and circadian pathways. Science. 2007;318:1786-1789.

18. Duez H, Staels B. The nuclear receptors Rev-erbs and RORs integrate circadian rhythms and metabolism. Diab Vasc Dis Res. 2008;5:82-88.

19. Delezie J, Dumont S, Dardente H, et al. The nuclear receptor REVERBalpha is required for the daily balance of carbohydrate and lipid metabolism. FASEB J. 2012;26:3321-3335.

20. Armstrong S. A chronometric approach to the study of feeding behavior. Neurosci Biobehav Rev. 1980;4:27-53.

21. Joly-Amado A, Cansell C, Denis RG, et al. The hypothalamic arcuate nucleus and the control of peripheral substrates. Best Pract Res Clin Endocrinol Metab. 2014;28:725-737.

22. Ueno $\mathrm{H}$, Nakazato $\mathrm{M}$. Mechanistic relationship between the vagal afferent pathway, central nervous system and peripheral organs in appetite regulation. J Diabetes Investig. 2016;7:812-818.

23. Patton DF, Mistlberger RE. Circadian adaptations to meal timing: neuroendocrine mechanisms. Front Neurosci. 2013;7:185.

24. Delezie J, Dumont S, Sandu C, Reibel S, Pevet P, Challet E. Reverbalpha in the brain is essential for circadian food entrainment. Sci Rep. 2016;6:29386.

25. Ayadi A, Birling MC, Bottomley J, et al. Mouse large-scale phenotyping initiatives: overview of the European Mouse Disease Clinic (EUMODIC) and of the Wellcome Trust Sanger Institute Mouse Genetics Project. Mamm Genome. 2012;23:600-610.

26. Paxinos G, Franklin KB. The Mouse Brain in Stereotaxic Coordinates. San Diego, CA: Academic Press; 2004.

27. Stengel A, Wang L, Goebel-Stengel M, Tache Y. Centrally injected kisspeptin reduces food intake by increasing meal intervals in mice. NeuroReport. 2011;22:253-257.

28. Giusti SA, Vercelli CA, Vogl AM, et al. Behavioral phenotyping of Nestin-Cre mice: implications for genetic mouse models of psychiatric disorders. J Psychiatr Res. 2014;55:87-95.

29. Kornmann B, Schaad O, Bujard H, Takahashi JS, Schibler U. Systemdriven and oscillator-dependent circadian transcription in mice with a conditionally active liver clock. PLoS Biol. 2007;5:e34.

30. Grosbellet E, Dumont S, Schuster-Klein C, et al. Leptin modulates the daily rhythmicity of blood glucose. Chronobiol Int. 2015;32:637-649.

31. Ahren B, Baldwin RM, Havel PJ. Pharmacokinetics of human leptin in mice and rhesus monkeys. Int J Obes Relat Metab Disord. 2000;24:1579-1585.

32. Briancon N, McNay DE, Maratos-Flier E, Flier JS. Combined neural inactivation of suppressor of cytokine signaling- 3 and protein-tyrosine phosphatase-1B reveals additive, synergistic, and factor-specific roles in the regulation of body energy balance. Diabetes. 2010;59:3074-3084.

33. Kennaway DJ, Varcoe TJ, Voultsios A, Boden MJ. Global loss of bmal1 expression alters adipose tissue hormones, gene expression and glucose metabolism. PLoS One. 2013;8:e65255.

34. Zhang Y, Fang B, Emmett MJ, et al. GENE, REGULATION. Discrete functions of nuclear receptor Rev-erbalpha couple metabolism to the clock. Science. 2015;348:1488-1492.

35. Shimba S, Ogawa T, Hitosugi $S$, et al. Deficient of a clock gene, brain and muscle Arnt-like protein-1 (BMAL1), induces dyslipidemia and ectopic fat formation. PLoS One. 2011;6:e25231.
36. Mang GM, La Spada F, Emmenegger Y, et al. Altered sleep homeostasis in Rev-erbalpha knockout mice. Sleep. 2016;39:589-601.

37. Vollmers C, Gill S, DiTacchio L, Pulivarthy SR, Le HD, Panda S. Time of feeding and the intrinsic circadian clock drive rhythms in hepatic gene expression. Proc Natl Acad Sci USA. 2009;106:21453-21458.

38. Bugge A, Feng D, Everett LJ, et al. Rev-erbalpha and Rev-erbbeta coordinately protect the circadian clock and normal metabolic function. Genes Dev. 2012;26:657-667.

39. Dahlstrand J, Lardelli M, Lendahl U. Nestin mRNA expression correlates with the central nervous system progenitor cell state in many, but not all, regions of developing central nervous system. Brain Res Dev Brain Res. 1995;84:109-129.

40. Solt LA, Wang Y, Banerjee S, et al. Regulation of circadian behaviour and metabolism by synthetic REV-ERB agonists. Nature. 2012;485:62-68.

41. Besharse JC, McMahon DG. The retina and other light-sensitive ocular clocks. J Biol Rhythms. 2016;31:223-243.

42. Plata-Salaman CR, Oomura Y. Food intake dependence on acute changes in light schedule. Physiol Behav. 1987;41:135-140.

43. Guastavino JM, Bertin R, Portet R. Effects of the rearing temperature on the temporal feeding pattern of the staggerer mutant mouse. Physiol Behav. 1991;49:405-409.

44. Turek FW, Joshu C, Kohsaka A, et al. Obesity and metabolic syndrome in circadian Clock mutant mice. Science. 2005;308:1043-1045.

45. Adamovich Y, Rousso-Noori L, Zwighaft Z, et al. Circadian clocks and feeding time regulate the oscillations and levels of hepatic triglycerides. Cell Metab. 2014;19:319-330.

46. Kettner NM, Mayo SA, Hua J, Lee C, Moore DD, Fu L. Circadian dysfunction induces leptin resistance in mice. Cell Metab. 2015;22:448-459.

47. Feillet CA, Bainier C, Mateo M, et al. Rev-erbalpha modulates the hypothalamic orexinergic system to influence pleasurable feeding behaviour in mice. Addict Biol. 2017;22:411-422.

48. Lu XY, Shieh KR, Kabbaj M, Barsh GS, Akil H, Watson SJ. Diurnal rhythm of agouti-related protein and its relation to corticosterone and food intake. Endocrinology. 2002;143:3905-3915.

49. Ellis C, Moar KM, Logie TJ, Ross AW, Morgan PJ, Mercer JG. Diurnal profiles of hypothalamic energy balance gene expression with photoperiod manipulation in the Siberian hamster, Phodopus sungorus. Am J Physiol Regul Integr Comp Physiol. 2008;294:R1148-R1153.

50. Wang D, Opperhuizen AL, Reznick J, et al. Effects of feeding time on daily rhythms of neuropeptide and clock gene expression in the rat hypothalamus. Brain Res. 2017;1671:93-101.

\section{SUPPORTING INFORMATION}

Additional Supporting Information may be found online in the supporting information tab for this article.

How to cite this article: Sen S, Dumont S, Sage-Ciocca D, et al. Expression of the clock gene Rev-erb $\alpha$ in the brain controls the circadian organisation of food intake and locomotor activity, but not daily variations of energy metabolism. J Neuroendocrinol 2018;30:e12557. https://doi.org/10.1111/jne.12557 